\title{
Retraction of: The speed of a biased random walk on a Galton-Watson tree is analytic*
}

\author{
Adam Bowditch $^{\dagger} \quad$ Yuki Tokushige ${ }^{\ddagger}$
}

\begin{abstract}
An erratum for the paper is given.

Keywords: Galton-Watson tree; biased random walks; speed; analytic.

MSC2020 subject classifications: Primary 60J80, Secondary 60K37; 60J10; 05C81.

Submitted to ECP on October 5, 2021, final version accepted on October 31, 2021.
\end{abstract}

The proofs of Theorem 1.1 and Lemma 3.1 use compact convergence of analytic functions to prove analyticity of the limiting function. Such an argument only holds for complex analytic functions and therefore the argument is insufficient to deduce the aforementioned results. 


\section{Electronic Journal of Probability Electronic Communications in Probability}

\section{Advantages of publishing in EJP-ECP}

- Very high standards

- Free for authors, free for readers

- Quick publication (no backlog)

- Secure publication $\left(\mathrm{LOCKSS}^{1}\right)$

- Easy interface (EJMS²)

\section{Economical model of EJP-ECP}

- Non profit, sponsored by $\mathrm{IMS}^{3}, \mathrm{BS}^{4}$, ProjectEuclid ${ }^{5}$

- Purely electronic

\section{Help keep the journal free and vigorous}

- Donate to the IMS open access fund ${ }^{6}$ (click here to donate!)

- Submit your best articles to EJP-ECP

- Choose EJP-ECP over for-profit journals

\footnotetext{
${ }^{1}$ LOCKSS: Lots of Copies Keep Stuff Safe http://www. lockss.org/

${ }^{2}$ EJMS: Electronic Journal Management System http://www.vtex.lt/en/ejms.html

${ }^{3}$ IMS: Institute of Mathematical Statistics http://www.imstat.org/

${ }^{4}$ BS: Bernoulli Society http://www. bernoulli-society.org/

${ }^{5}$ Project Euclid: https://projecteuclid.org/

${ }^{6}$ IMS Open Access Fund: http://www.imstat.org/publications/open.htm
} 